\title{
Evidence-based landscape architecture: The maturing of a profession
}

Robert D. Brown, Robert C. Corry

NOTICE: This is the authors' version of a work that was accepted for publication in LANDSCAPE AND URBAN PLANNING: AN INTERNATIONAL JOURNAL OF

LANDSCAPE ECOLOGY, PLANNING AND DESIGN. Changes resulting from the publishing process, such as peer review, editing, corrections, structural formatting, and other quality control mechanisms, may not be reflected in this document. Changes may have been made to this work since it was submitted for publication. A definitive version was subsequently published in LANDSCAPE AND URBAN PLANNING: AN INTERNATIONAL JOURNAL OF LANDSCAPE ECOLOGY, PLANNING AND DESIGN, VOLUME 100, ISSUE 1, March 1, 2011. If you refer to this work please cite the published version. 


\begin{abstract}
Professions tend to change over time, typically becoming more scholarly as information is generated and incorporated into practice. Medicine has made the transition to a scholarly profession over the past hundred years or so and has recently embraced the concept of evidencebased medicine. Landscape architecture is poised to become a more scholarly profession and this essay proposes that it become a discipline of evidence-based landscape architecture. Actions for everyone from professors and administrators to practicing professionals are suggested to meet the goal of a more scholarly, relevant profession. The risk of ignoring the trend of evidence as a basis for practice is described in terms of the divergence among historical medical disciplines.
\end{abstract}


Medical practice used to be largely based on four "humors": black bile, yellow bile, phlegm, and blood. Treatments were intended to expel a harmful surplus of one of the humors through actions such as blood-letting and purging, practices that were often not very effective. This theoretical foundation "dominated medical thinking well into the seventeenth century" (Atkins, 1982, p. 284) and did not completely collapse until the mid-1800s (Voswinckel, 2000). It is possible that physicians treating George Washington for a throat infection killed him through well-intentioned bloodletting (Cheatham, 2008).

Medicine has since transitioned from these humble beginnings (Starr, 1982) to its current position as a powerful, scholarly discipline practicing "evidence-based medicine" or EBM(e.g., Rosenberg and Donald, 1995). Rather than relying only on what they learned in medical school and through their professional practice, physicians now base decisions on the best available factual evidence that has been generated through scholarly research. The medical discipline is probably the most prolific in terms of methodically reporting on trials and treatments, yet it is medical practice that engages the public and sustains human health.

A tongue-in-cheek article in the British Medical Journal described how clinical decisions are made when EBM is not available. The methods ranged from "eminence-based medicine," where a person's reputation as an expert transcends evidence, to "eloquence-based medicine," where fancy suits and verbal acuity are more powerful than evidence (Isaacs and Fitzgerald, 1999). While the article's facetious tone was aimed at medical practitioners, such characteristics can be found in professionals of all fields.

Steiner (2002) characterized some of the most recent trends in landscape architecture from a period when landscape architecture "retreated behind the garden wall" (p. 91) in the mid 1900 s to when the discipline decisively returned to its roots in the 1960s and 1970s, only to retreat again in the 1980s. He criticized the profession for engaging in destructive development and superficial, glitzy design as it transitioned through the eloquence- and eminence-based phases, yet he noted the profession had recently moved toward a stronger theoretical foundation, and by recommendation encouraged this trend.

Despite the recommendation from Steiner and other academics, much of contemporary practice in landscape architecture is still based on beliefs rather than facts. This is due at least in part to the dearth of factual information upon which to base decisions. Both the profession and the discipline of landscape architecture have a culture of non-reporting. There are very few articles in the scholarly literature that evaluate the effectiveness of completed projects. Built landscapes are seldom tested or monitored to see if they achieved their stated objectives, and thus mistakes are repeated with remarkable and embarrassing efficiency. Landscape architects implicitly derive new hypotheses for every project, yet seldom think of built projects as field experiments, rarely observe and record the effects to see if the design objectives were met, and almost never report back to the discipline (Ahern, 1999). What results is a practice growing in small increments with myriad missed opportunities to further the documented evidence that could support better-informed design. Said another way: if current landscape architecture were likened to medical practice we might still be practicing blood-letting and the prognosis for healing the landscape through design would be bleak.

It is not much better in the academy. Gobster et al. (2010) noted that the amount of research published in peer-reviewed journals remains dismally low among landscape architecture faculty. In their sample of 286 full-time North American faculty members they found that only 6\% (17 individuals) published one or more peer-reviewed papers each year and that nearly half of the faculty members they searched published no peer-reviewed papers in a ten-year period. 
Again comparing the scholarship of landscape architecture with medicine, at our current rate of published evidence we are doing little to prevent our landscapes from sliding toward states of morbidity. Soil erosion, impaired water quality, degraded streams, aquatic dead zones, biodiversity loss and extirpation and extinction of species, necrosis and premature death of (especially city) plants, contagion of disease or pests, and intolerable urban living conditions (unless subsidized by fossil-fuel sponsored comfort) are all indicative of unhealthy or unhealthful landscapes. While landscape architects are not solely responsible for any one of these, we must ask ourselves: to what extent do our designs and plans prevent or solve these problems rather than contribute to them?

Medicine has become one of the most powerful and respected professions by embracing scholarly information, methodical record keeping, monitoring, and reporting. These approaches are instructive for landscape architecture. We suggest that it is time for the profession to transition to "evidence-based landscape architecture," or EBLA. EBLA has not yet been defined so we propose the following definition: evidence based landscape architecture is the deliberate and explicit use of scholarly evidence in making decisions about the use and shaping of land. EBLA supports decisions but does not dictate them (e.g., Fisher, 2004), and it uses knowledge generally from methodically studied experiment or experience - as the principal information source for design.

Distinguishing between the known facts and information and the yet-unknown yields cautious but fundamentally sound design decision-making. This leads to acknowledging the limits of knowledge in the process of design and more clearly articulates the product of design as the embodiment of hypotheses. To employ EBLA as an approach requires that we ask, "Do we know enough to act?" (Smiley, 1997, p. 42). In her admonition of distinguishing and using knowledge over beliefs (Smiley, 1997) cautions, "As. . .our population grows, the margin one is allowed for survival by the abundance of nature and the expanse of geography grows smaller and smaller. Mistakes, even sincere mistakes of sincere belief, have ever more serious consequences" (p. 42).

The analogy with medicine can be taken further. The landscape is a patient and the landscape architect the physician. Several leading landscape architectural thinkers use "landscape health" as a powerful metaphor (see Steiner, 2002, p. 89). Hippocrates' rule of "do no harm" for human patients is applicable to the landscape.

Some of the difficulties medicine has faced in implementing EBM can be instructive. There will almost certainly be resistance, most likely from those practicing expert-based landscape architecture (which is distinctly different from expertise-based landscape architecture), but younger practitioners will likely embrace it enthusiastically as it relieves some of the illdefined eminence of design and provides a sound footing. EBM has been criticized for the following: being too narrow in what it defines as evidence, unwarranted extrapolations of laboratory results to the real world, and lack of appropriate communication to practitioners. These problems can be avoided in EBLA by addressing them explicitly from the start. Milburn et al. (2003) have provided a framework for identifying and assessing the scholarliness of landscape architectural enterprises. This could be expanded upon to illustrate how these criteria would be articulated in the various components of landscape architecture.

Landscape architecture is far more than just design, and EBLA needs to be more than evidencebased design. There are several areas of landscape architecture that already have a strong evidence base-areas like soil drainage, transplanting of trees, microclimate modification, materials engineering, and visual preference. In fact, this encompasses many of the physical, 
several of the biological, and a few of the human components of the landscape. These models should be extended and applied to other areas such as social and cultural components, aesthetics, and concepts like genius loci [note the eminence of Latin].

Academic landscape architects need to both generate more scholarly information that will inform decision-making and communicate it to practitioners in a way that can be readily applied. Physicians regularly access journal articles in support of their medical practice. Journals like Landscape and Urban Planning could provide such a resource for landscape architects, particularly if the articles published address issues of interest to practitioners. Practitioners interested in using research results and methodically learned evidence as a basis for design would need to know where to look, what to look for, and how to apply it: while novel in the context of EBLA, these form the foundation of design interventions. Further, practitioners need a reasonable model for developing their own reporting of evidence in design. While they can look to a very small number of consistently publishing academics, they would not find on aggregate that there is a compelling reason to share evidence, nor a norm for how or where to report it. A small cadre of practitioners write in Landscape Architecture magazine (LAM); this begins to demonstrate how practice reviews might translate to evidence. Better yet is the periodic LAM reporting on research of interest to practitioners - a way to get some of the most salient research results without going to the nearest university library.

Landscape architecture is at a stage similar to medicine when antibiotics were invented. Many people were dying every year from infections and the new medicines provided instant relief. There are issues of similar import in the world today - global climate change, urban heat islands, mass migration to urban areas, loss of biological diversity - some of which are fatal to people and other organisms. Landscape architecture has a role to play in addressing these issues and many more. But our recommendations cannot be based on belief. They must be based on facts and knowledge: evidence.

Rosenberg and Donald (1995) proposed a four-step process by which physicians can practice EBM. There are similarities between these four steps and the characteristics of scholarly contributions (Milburn et al., 2003) suggesting the following process for EBLA: (1) formulate a clear goal or question based on the issue or the client's request (e.g., extend the season of use of an outdoor area); (2) search for relevant scholarly literature; (3) critically evaluate the evidence for both validity and usefulness; and (4) synthesize and apply the findings to the problem at hand.

Some academic landscape architects need to focus their scholarly activities on basic research to identify the underlying causes, effects, and possible solutions to important issues. At the same time there is the need for others to conduct meta-analyses of information published in other relevant disciplines, including landscape and urban planning, and synthesize the information into a format that is understandable and useful to practitioners. Academic administrators should focus their attention on convincing funding agencies to provide research funds specifically for landscape architectural research. Professors need to develop and teach their students processes for accessing and using evidence as a basis for their design projects (design boards should cite evidence!). There needs to be a forum for practitioners to inform academics of their research needs and report their own methodically-gathered evidence. Finally, there is a need for publications like Landscape and Urban Planning to provide a vehicle for circulating hardearned knowledge among academics, practitioners, and the public.

Landscape architecture should resist the temptation to have experts in different aspects of the field unless those experts are basing their expertise on factual evidence. Physicians who became 
experts through their clinical practice made major errors that cost many lives. For example, cardiac experts long recommended that heart attack survivors stay in bed for several weeks, during which time many died from blood clots in their lungs.

Landscape architecture has the potential to be as important to the health and well-being of the landscape and the populations in it as medicine is to humanity. It likewise has the vulnerability to diminish in importance by ignoring the lessons of other disciplines.

A final story from the history of medicine merits consideration. In medieval times barbers and surgeons were one profession. Practitioners would cut your hair and trim your beard, but they would also perform surgery in the form of blood-letting, tooth extraction, and baby delivery. The familiar red, blue, and white barber's pole is variously attributed to bloody bandages wrapped around a pole or the red and blue umbilical cord that the barber/surgeon would wrap around the pole for parents after a birth. The gap between the practice of barbers and surgeons today is immense but historically narrow. Using evidence to plan, design, and manage the landscape is necessary to re-establish landscape architecture's respect, credibility, and leadership. If landscape architects do not use EBLA, then another profession will. If that happens, we will retreat behind the garden wall once again.

\section{Acknowledgements}

We appreciate the thoughtful comments and suggestions from the reviewers and the editor that greatly improved this paper. Thanks also to two transdisciplinary reviewers for their comments on an earlier version of this manuscript. 


\section{References}

Ahern, J., 1999. Spatial concepts, planning strategies and future scenarios: a framework method for integrating landscape ecology and landscape planning. In: Klopatek, J.M., Gardner, R.H. (Eds.), Landscape Ecological Analysis: Issues and Applications. Springer-Verlag, New York, pp. 175-201.

Atkins, E., 1982. Fever-its history, cause, and function. Yale J. Biol. Med. 55, 283- 289.

Cheatham,M.L., 2008. The death of George Washington: an end to the controversy? Am. Surgeon $74,770-774$.

Fisher, T., 2004. Architects behaving badly. Harvard Des. Mag. 21, 1-3.

Gobster, P.H., Nassauer, J.I., Nadenicek, D.J., 2010. Landscape journal and scholarship in landscape architecture: the next 25 years. Landscape J. 29, 52- 70.

Isaacs, D., Fitzgerald, D., 1999. Seven alternatives to evidence based medicine. Br. Med. J. 319, 1618.

Milburn, L.A.S., Brown, R.D., Mulley, S.J., Hilts, S.G., 2003. Assessing academic contributions in landscape architecture. Landscape Urban Plan. 64, 119- 129.

Rosenberg, W., Donald, A., 1995. Evidence based medicine - an approach to clinical problem solving. Br. Med. J. 310 (6987), 1122-1126.

Smiley, J., 1997. Farming and the landscape. In: Nassauer, J.I. (Ed.), Placing Nature: Culture and Landscape Ecology. Island Press, Washington, DC, pp. 33- 43.

Starr, P., 1982. The Social Transformation of American Medicine. Basic Books, New York.

Steiner, F.R., 2002. Human Ecology: Following Nature's Lead. Island Press, Washington, DC.

Voswinckel, P., 2000. From uroscopy to urinalysis. Clin. Chim. Acta 297, 5-16. 